\title{
MICROHABITATS DE MOSQUITOS (DIPTERA, CULICIDAE) EM INTERNÓDIOS DE TAQUARA NA MATA ATLÂNTICA, PARANÁ, BRASIL
}

\author{
Ana Leuch Lozovei ${ }^{1}$
}

\begin{abstract}
MOSQUITOES MICROHABITATS (DIPTERA, CULICIDAE) IN BAMBOO INTERNODES IN ATLANTIC FOREST, PARANÁ, BRAZIL. During two consecutive years, from January 1985 to December 1986, a comparative study of mosquitoes preferences for breeding habitat was carried out in the Atlantic Forest of the Serra do Mar, Paraná State, Brazil. To achieve it, 1875 bamboo internodes aligned vertically in live green, bamboo plants Merostachys speciosa Munro and Merostachys sp. were used, in which metabolic water was exuded from the plant itself, and presenting different size/pattern holes at their lateral walls, bored by the local sylvan fauna. Another group of 1200 individual internode traps was used as comparative element, carved out with a transversal cut by a saw, filled with local stream water and held in branches at different heights in the vegetal strata nearby. At both microhabitat types, a total of 17 culicid species was registered. Culex (Microculex) neglectus Lutz, 1904, Cx. (Carrollia) soperi Antunes \& Lane, 1937, Sabethes (Sabethes) batesi Lane \& Cerqueira, 1942 and Sa. (Sabethinus) melanonymphe (Dyar, 1924) colonized exclusively live plant internodes, while Culex (Microculex) elongatus Rozeboom \& Lane, 1950, Cx. (Carrollia) iridescens (Lutz, 1905), Cx. (Carrollia) kompi Valencia, 1973 and Trichoprosopon (Trichoprosopon) soaresi Dyar \& Knab, 1907 bred only in internode traps. The remaining nine species colonized both habitats indistinctly. Quantitatively, was detected the abundance of $60.1 \%$ at live green internodes, against $39.9 \%$ for internode traps. Concerning the different patterns of bored live internode holes, $40.3 \%$ of the total computed specimens were collected in square or rectangular holes, $31.9 \%$ in two hole internodes, one minute circular, the other wider, and the remaining $28.8 \%$ of specimens distributed in other pattern type internodes. The mosquitoes breeding at these microhabitats fall in the culicid entomofauna specialized at locating and detecting peculiar and propitious mesogen conditions for breeding purposes.
\end{abstract}

KEYWORDS. Culicidae, reproduction, bamboo internodes, microhabitats.

\section{INTRODUÇÃO}

A entomofauna culicidiana procria-se em uma grande variedade de criadouros de diferentes dimensões e volumes de água. Algumas espécies têm considerável plasticidade de adaptação aos locais utilizados na sua procriação e ocorrem em diferentes

1. Departamento de Patologia Básica, Setor de Ciências Biológicas da Universidade Federal do Paraná, Centro Politécnico, Caixa Postal 19031, CEP 81531-990, Curitiba, Paraná, Brasil. (alozovei@bio.ufpr.br) 
tipos de habitats. Outras, ao contrário, são mais restritas na escolha de seus criadouros (SERvice, 1976). No Japão, NAKata et al. (1953) estudaram larvas que passam o inverno em diapausa; MoriYa (1974) estudou a distribuição sazonal das espécies dendrícolas com auxílio de ovitrampas feitas de internódios de bambu. Em Sri Lanka, AmERAsINGUE \& Alagoda (1984) verificaram a estratificação vertical de sete espécies dendrícolas. Na América do Norte, WALKER et al. (1991) trabalharam com a interação dos processos biótico e fatores físico-químicos da água em ocos de árvores.

No Brasil, há algumas referências, como as de Lozover \& Luz (1976), Gomes et al. (1992), Lopes (1997), Lozover (1998) e Silva \& Lozovei (1999), sobre mosquitos que se procriam em internódios de taquara artificialmente instalados e em habitats similares, como em ocos de árvores. Espécies de mosquitos silvícolas dendrotelmatas estudadas têm seu desenvolvimento restringido a determinados tipos de criadouros e se procriam em recipientes naturais formados por internódios de taquara, planta típica dos trópicos e subtrópicos do mundo, constituindo habitats de pequeno porte.

Objetiva-se averiguar a abundância e a preferência das espécies de culicídeos em internódios de taquara-verde, enfileirados e inseridos naturalmente nos colmos em pé, com diferentes combinações de dimensões e padrões de furos nas paredes laterais, brocados pela fauna local, e comparar com os internódios-armadilhas afixados em galhos vegetais em estratos verticais.

\section{MATERIAL E MÉTODOS}

A investigação foi desenvolvida no interior da Mata Atlântica, Serra do Mar, Município de Quatro Barras, $25^{\circ} 19^{\prime} 55^{\prime \prime} \mathrm{S}, 48^{\circ} 54^{\prime} 35^{\prime \prime} \mathrm{W}$ e altitude de $974 \mathrm{~m}$, Estado do Paraná, em área localizada na altura do km 33 da Rodovia Estadual 410, conhecida por Estrada da Graciosa. Na Serra do Mar, resta a última reserva de matas virgens da Floresta Atlântica, entremeada por vegetação rasteira, árvores de pequeno e médio portes e algumas que alcançam até $50 \mathrm{~m}$ de altura. Em contraste com a diversificada cobertura vegetal, crescem em grande quantidade gramíneas, como taquara (Merostachys sp.), taquara-poca (Merostachys speciosa Munro), taquarinha (Olyra glaberrima Raddi) e criciúma (Chusquea sp.), que em determinadas áreas tornam o matagal quase intransitável (MAACK, 1981).

Foram avaliados internódios de colmos de taquara Merostachys speciosa e Merostachys sp. de 200 a $400 \mathrm{~mm}$ de comprimento, estratificados naturalmente nos colmos de 0 a $15 \mathrm{~m}$ de altura que, normalmente, em suas paredes laterais continham furos de diferentes padrões e dimensões, elaborados por fauna silvícola. Os furos circulares apresentavam-se de $2 \mathrm{~mm}$ e de 5 a $8 \mathrm{~mm}$ de diâmetro, enquanto os quadrados e retangulares ostentavam as aberturas com grande gama de variações, como $5 \times 5$ a 10x10, $8 \times 15$ a $25 \times 40 \mathrm{~mm}$ (fig. 1). A água acumulada no interior dos internódios verdes provém do próprio metabolismo dos colmos, o que contribui para formar criadouros para determinadas espécies de mosquitos. Para obter elementos de comparação, concomitantemente, foram estudados internódios-armadilhas individuais, com água de rio adicionada, instalados em árvores ao lado do taquaral em alturas de 0 a $4 \mathrm{~m}$. Seus comprimentos variavam de 300 a $400 \mathrm{~mm}$ e as aberturas no corte transversal, de 40 a 50 $\mathrm{mm}$ de diâmetro. A estratificação vertical das amostras foi mencionada apenas para indicar o critério de amostragem. As coletas mensais de amostras, em ambas as modalidades de internódios, foram efetuadas durante dois anos consecutivos, de janeiro de 1985 a dezembro de 1986.

Para ter acesso às amostras de mosquitos em internódios de colmos verdes, foi necessário abater e abri-los um por um, recolhendo o seu conteúdo aquático juntamente com mosquitos imaturos (larvas e pupas). Ao mesmo tempo, foram conferidas as particularidades, como o número, a forma e o tamanho de furos isolados ou associados nas paredes laterais. Foram analisadas amostras de mosquitos de cada um dos internódios que apresentavam as características preestabelecidas. As amostras de mosquitos foram depositadas na "Coleção Pe. Jesus Moure", Departamento de Zoologia, Universidade Federal do Paraná, Curitiba (DZUP). A entomofauna associada nestes microhabitats, formigas, foi identificada pelo Dr. Carlos Roberto Ferreira Brandão, Museu de Zoologia, Universidade de São Paulo, São Paulo, sendo parte dos espécimens depositada nesse Museu (MZSP).

Em laboratório, as amostras de mosquitos imaturos foram mantidas individualmente, até que atingissem o $4^{\circ}$ estádio larvar e uma grande parte, até a fase de adulto, utilizada posteriormente para a identificação das 


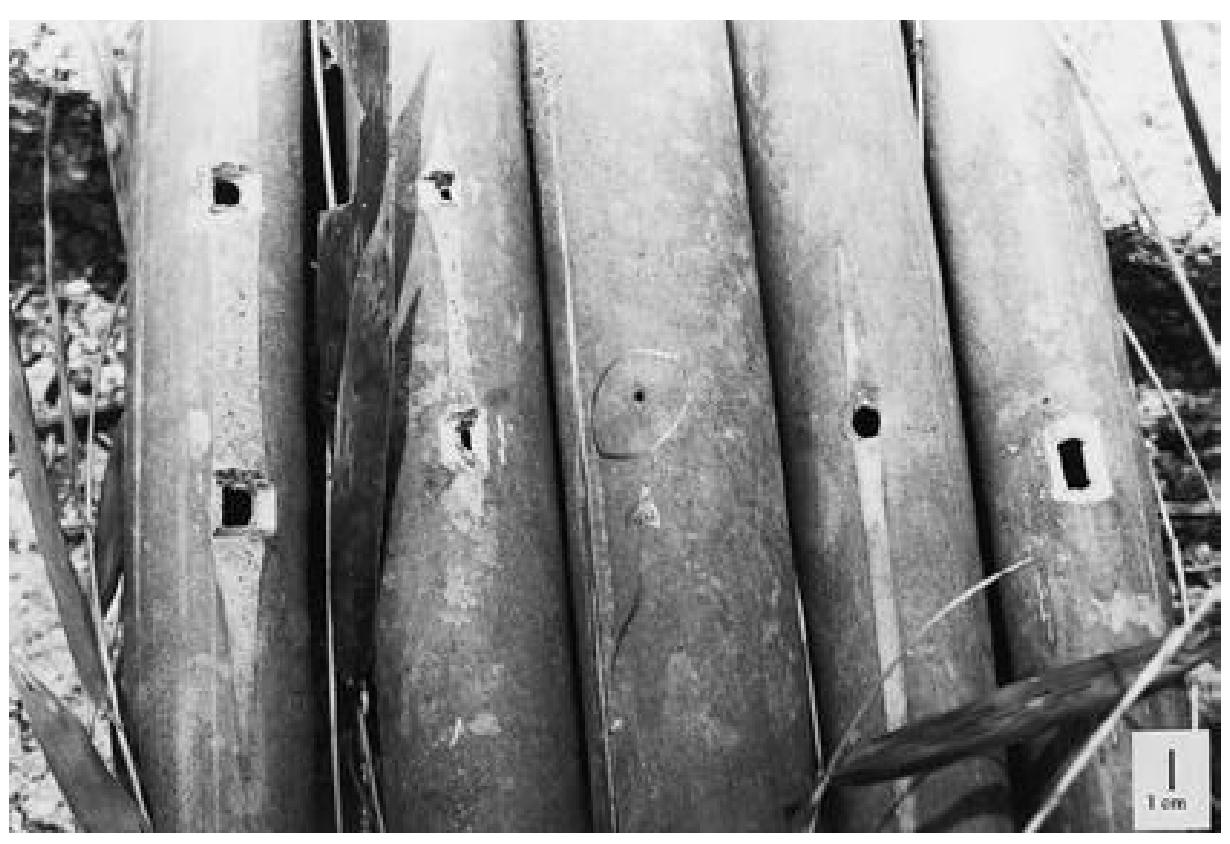

Fig. 1. Internódios de taquara verde, Merostachys sp. com diferentes padrões e dimensões de furos elaborados pela fauna silvícola da Mata Atlântica, Paraná, Brasil.

espécies. Para identificar os culicídeos dendrícolas, foram empregadas chaves de ForATTINI (1965a, 1965b) e LANE (1953a, 1953b). Mais detalhes da metodologia empregada no campo e no laboratório e de outros dados podem ser obtidos em Lozover (1998).

\section{RESULTADOS E DISCUSSÃO}

Entre as 17 espécies de mosquitos dendrícolas estudadas, treze ocorreram em internódios verdes com água metabólica exsudada da própria planta, que se apresentavam com diferentes padrões e dimensões de furos nas paredes laterais, brocados pela fauna local. A pesquisa resultou de 120 colmos abatidos com 1.875 internódios analisados, $1.350(72,0 \%)$ dos quais foram positivos para mosquitos e colonizados por 3.725 imaturos, $242(12,9 \%)$, negativos e $283(15,1 \%)$, sem furos e, por conseguinte, também negativos para esta entomofauna. Em internódios-armadilhas, dos 1.200 instalados, $1.019(84,9 \%)$ apresentaram-se positivos, ocupados por 2.477 exemplares de culicídeos, enquanto 181 (15,1\%), foram negativos. Estes microhabitats foram colonizados também por treze espécies de mosquitos, nove das quais coincidiram com as espécies encontradas em internódios verdes (tab. I).

O número de mosquitos imaturos por internódio de taquara parece não depender do volume de água. Em internódios de taquara verde, a quantidade de água oscilou de 10 a $300 \mathrm{ml}$, enquanto em internódios-armadilhas, variou de 100 a $500 \mathrm{ml}$. Ao longo da investigação, foi observado que em pequenos volumes de água, (10 a $100 \mathrm{ml})$ havia de 
1 a 5 imaturos por internódio. Entretanto, esporadicamente, foi verificada a presença de muitos (15 a 20) exemplares de larvas não predadoras por internódio. Praticamente o mesmo fenômeno repetiu-se em internódios-armadilhas, onde havia água em volumes bem maiores. Isto sugere, portanto, que a quantidade destes mosquitos por microhabitat dendrícola não foi proporcional ao volume de água.

A distribuição de mosquitos não foi uniforme nos hidrobiótopos estudados. Houve internódios com muitos indivíduos, com poucos e até sem nenhum. O número médio de mosquitos imaturos por internódio-verde foi muito variado, oscilou de 1,0 a 8,3 exemplares. Em internódios-armadilhas, esta variação manteve de 1,1 a 4,0 exemplares por internódio, cerca de 50\% menor do que a anterior. Às espécies, que colonizaram internódios- verdes, coube a maior abundância de imaturos por internódio (tab. I).

Considerando os internódios retirados de colmos verdes com diferentes combinações de furos para verificar a abundância de cada espécie por recipiente, observase que Wyeomyia (Wyeomyia) lutzi (Costa Lima, 1930) distinguiu-se com maior média de 8,3 imaturos em internódios com furos quadrados ou retangulares, com 5,6 em internódios com furos circulares diminutos, com 5,4 indivíduos em internódios com dois furos circulares e com 4,0 imaturos em internódios com três furos (dois circulares e um quadrado ou retangular). Em seqüência, Wy. (Dendromyia) personata (Lutz,1904) destacou-se com 6,0 espécimens em internódios de furo quadrado ou retangular, com 4,5 exemplares em internódios com três furos (dois circulares e um quadrado ou retangular). Nos demais internódios, a abundância oscilou de 3,5 a 3,8. Wy. (Wyeomyia) limai Lane \& Cerqueira, 1942, também se destacou, colonizando em maior abundância na proporção de 5,4 imaturos os internódios de três furos (dois circulares e um quadrado ou retangular), com 5,3 indivíduos os de dois furos (um circular e um quadrado ou retangular) e, ainda, com 4,0 imaturos os de um furo (quadrado ou retangular). Em outros microhabitats, variou de 2,4 a 2,8. Parece que estas três espécies não-predadoras apresentaram maior número médio de imaturos por internódio por dois motivos. Primeiro, por terem maior adaptabilidade nestes microhabitats, ocorrendo tanto em internódios com água límpida, como em internódios com água poluída e matéria orgânica em decomposição. Segundo, neste tipo de habitat, não ocorreu superposição de larvas predadoras, a não ser eventualmente com as de Toxorhynchites (Lynchiella) bambusicola (Lutz \& Neiva, 1913) e To. (Lynchiella) pusillus (Costa Lima, 1931). Vale destacar uma outra característica destas três espécies, a ocorrência de muitos exemplares (15 a 20) num mesmo internódio com muito sedimento e sem superposição. Foi isso que lhes elevou o número médio de indivíduos por internódio.

Outras duas espécies, que colonizaram internódios de taquara-verde com significativo número médio de imaturos, foram: Culex (Microculex) neglectus Lutz, 1904 em internódios de um furo quadrado ou retangular com 6,0 imaturos, em internódios de três furos (dois circulares e um quadrado ou retangular) com 4,5 imaturos e, ainda, em internódios de dois furos (um circular e quadrado ou retangular) com 4,0 exemplares; Cx. (Carrollia) soperi Antunes \& Lane, 1937 com 4,0 indivíduos em internódios de dois furos (um circular e um quadrado ou retangular). Nos outros dois padrões de furos, a oscilação foi de 1,8 e 3,0 indivíduos por internódio. Estas duas espécies diferenciamse das três acima nominadas por terem sido encontradas com muitos indivíduos em número reduzido de internódios e sem superposição com predadoras. 
As demais oito espécies ocorridas em internódios verdes tiveram número médio de imaturos de 1,0 a 3,7 exemplares, colonizando internódios em superposição com um ou mais predadores. Isto, talvez, constitua um dos fatores do pequeno número de exemplares por internódio.

Considerando a dispersão das diferentes espécies que colonizaram internódios retirados de colmos verdes, o coeficiente de variação oscilou de 66,7\% em internódios de dois furos a $128,5 \%$ em internódios de um furo circular diminuto. As variações intermediárias de $81,8 \%$ - 94,1\% - 96,0\% ocorreram em internódios de outras combinações de furos. Já em internódios-armadilhas, o coeficiente de variação foi de $73,7 \%$. Percebe-se claramente que os mosquitos não se procriaram uniformemente em todos os microhabitats. Os internódios com furos circulares diminutos (de $2 \mathrm{~mm}$ de diâmetro) foram acessíveis somente a oito espécies. As que ocorreram neste tipo de microhabitat também fizeram-se presentes em internódios com outros padrões de furos. São espécies que possuem mais ampla adaptabilidade a estes habitats dendrotélmicos em contraposição às outras nove, que se procriaram somente em internódios com furos mais amplos (tab. I).

Paralelamente, confrontando a dispersão das espécies em ambos os microhabitats, em internódios-verdes colonizados por treze espécies, a dispersão foi de $16,1 \%$ a $107,1 \%$, enquanto em internódios-armadilhas,colonizados também por treze espécies, o coeficiente de variação manteve-se entre $15,6 \%$ e $92,8 \%$ (tab. I).

As 17 populações desta entomofauna culicidiana são formadas por oito espécies de larvas predadoras e canibais e de nove espécies não predadoras. Normalmente, estes dois grupos de larvas procriaram-se em superposição, com exceção de apenas poucos internódios em que não foi constatada a coexistência. A ação predatória e canibal exerce um certo controle das populações por microhabitat. Nos microhabitats com superposição de predadores, o número médio de imaturos por internódio oscilou de 1 a 5 indivíduos. A coexistência num mesmo internódio ocorreu entre duas ou mais espécies em diferentes combinações. Entre as sintopias mais comuns e frequientes, de predador com presa, foram nas proporções de $1: 1,1: 2,1: 3,1: 4$, ou ainda 2:2, 2:3, 2:4. Também houve outras proporções de predador $\mathbf{x}$ presa. As sobreposições abrangeram diferentes estádios larvares, tanto de predadores como de não predadores e, mesmo, entre as espécies predadoras. A análise detalhada da superposição entre estes tipos diferentes de comportamentos na exploração de alimentos, é bastante complexa, pois envolve muitas variáveis biológicas e mesológicas. Saliente-se que predação e canibalismo constituem um dos fatores que reduzem o número de imaturos nestes microhabitats. Há outros fatores que influenciam esta dinâmica numérica. Podem ser endógenos (genéticos), peculiares destas espécies de produzirem proles reduzidas ou, mesológicos, próprios das condições adversas destes criadouros. Devido à predação e canibalismo, as fêmeas talvez se utilizem de estratégia de oviposições parceladas, isto é, de oviporem pouco em diferentes internódios para garantir a sobrevivência da prole. Entretanto, houve exceções. Algumas espécies de não predadores ocorreram com número de imaturos bem mais elevado que o comum, isto é, de 15 a 20 indivíduos por internódio e sem superposição de predadores. A ausência de sintopias elevou o número médio de indivíduos por internódio entre 5 e 8 (tab. I). Esta abundância foi constatada em internódios em que houve muito sedimento, tanto em internódios-verdes, como em internódios-armadilhas. Nos internódios retirados de colmos verdes, o sedimento consistiu de fragmentos de 
Tabela I. Número médio de mosquitos imaturos (Culicidae) por internódios-verdes $(n=1875)$ abatidos de colmos verdes $(\mathrm{n}=120)$ com diferentes padrões e dimensões de furos*: 1350 positivos para mosquitos e colonizados por 3725 imaturos, 242 negativos para entomofauna e 283 sem furos; internódios-armadilhas instalados artificialmente $(\mathrm{n}=1200)$ com amplos furos circulares de corte transversal ${ }^{* *}: 1019$ positivos para mosquitos e colonizados por 2447 imaturos e 181 negativos para mosquitos. Mata Atlântica, Serra do Mar, Paraná, Brasil, período de janeiro 1985 a dezembro 1986. (c, furos circulares de 40-50 mm em internódios-armadilhas; em internódios de taquara verde: $\mathrm{cp}$, furos circulares de $2 \mathrm{~mm}$; cg, furos circulares de $5-8 \mathrm{~mm}$; q, furos quadrados $5 \times 5$ $\mathrm{mm}$ a $10 \times 10 \mathrm{~mm}$; $r$, furos retangulares de $8 \times 15 \mathrm{~mm}$ a 25x40 mm; p, larvas predadoras ou canibais).

\begin{tabular}{|c|c|c|c|c|c|c|c|c|}
\hline $\begin{array}{l}\text { Combinação de padrões } \\
\text { Dimensões de furos } \\
\text { Imaturos }\end{array}$ & $*_{\mathrm{cp}}$ & $\begin{array}{l}* \mathrm{cp} \\
* \mathrm{cg}\end{array}$ & $\begin{array}{l}* \mathrm{cp} \\
*_{\mathrm{q}}\end{array}$ & $\begin{array}{c}* \mathrm{cp}-\mathrm{cg} \\
* \mathrm{q} \\
* \mathrm{r}\end{array}$ & $\begin{array}{l}* \mathrm{q} \\
* \mathrm{r} \\
* \mathrm{r}\end{array}$ & $* \mathrm{CV}(\%)$ & $* * \mathrm{c}$ & $\begin{array}{c}\mathrm{CV}(\%) \\
\text { ambos habit }\end{array}$ \\
\hline Culex elongatus & 0,0 & 0,0 & 0,0 & 0,0 & 0,0 & - & 2,3 & - \\
\hline Cx. iridescens & 0,0 & 0,0 & 0,0 & 0,0 & 0,0 & - & 3,0 & - \\
\hline Cx. kompi & 0,0 & 0,0 & 0,0 & 0,0 & 0,0 & - & 2,1 & - \\
\hline Cx. neglectus & 0,0 & 0,0 & 4,0 & 4,5 & 6,0 & 93,1 & - & - \\
\hline Cx. soperi & 0,0 & 0,0 & 4,0 & 3,0 & 1,8 & 100,0 & 0,0 & - \\
\hline Sabethes aurescens (p) & 1,6 & 2,9 & 3,7 & 3,2 & 3,3 & 27,6 & 2,9 & 24,1 \\
\hline Sa. batesi $(\mathrm{p})$ & 1,6 & 1,0 & 2,0 & 1,5 & 1,0 & 28,6 & 0,0 & - \\
\hline Sa. melanonynphe $(\mathrm{p})$ & 2,8 & 2,3 & 3,0 & 1,0 & 2,9 & 33,3 & 0,0 & - \\
\hline Shannoniana fluviatilis & 3,7 & 3,3 & 2,5 & 3,3 & 2,7 & 16,1 & 3,6 & 15,6 \\
\hline Toxorhynchites bambusicola $(\mathrm{p})$ & 0,0 & 2,9 & 2,0 & 2,3 & 1,4 & 64,7 & 1,1 & 62,5 \\
\hline To. pusillus $(\mathrm{p})$ & 0,0 & 1,0 & 1,5 & 3,0 & 1,7 & 78,6 & 1,2 & 64,3 \\
\hline Trichoprosopon compressum (p) & 0,0 & 2,3 & 3,5 & 0,0 & 1,2 & 107,1 & 1,4 & 92,8 \\
\hline Tr. pallidiventer $(\mathrm{p})$ & 3,1 & 1,0 & 2,0 & 1,2 & 2,3 & 42,1 & 2,0 & 42,1 \\
\hline Tr. soaresi $(\mathrm{p})$ & 0,0 & 0,0 & 0,0 & 0,0 & 0,0 & - & 1,7 & - \\
\hline Wyeomyia limai & 2,4 & 2,8 & 5,3 & 5,4 & 4,0 & 35,9 & 4,0 & 30,8 \\
\hline Wy. lutzi & 5,6 & 5,4 & 3,7 & 4,0 & 8,3 & 33,3 & 3,2 & 38,0 \\
\hline Wy. personata & 3,8 & 3,5 & 3,5 & 4,5 & 6,0 & 23,2 & 3,8 & 21,4 \\
\hline Somatória por tipo de furo & 24,6 & 28,4 & 40,7 & 36,9 & 42,6 & - & 32,3 & - \\
\hline Média /internódio / padrão furo & 1,4 & 1,7 & 2,4 & 2,2 & 2,5 & - & 1,9 & - \\
\hline Desvio padrão (s) & 1,8 & 1,6 & 1,6 & 1,8 & 2,4 & - & 1,4 & - \\
\hline Coeficiente variação \% (C.V.) & 128,5 & 94,1 & 66,7 & 81,8 & 96,0 & - & 73,7 & - \\
\hline
\end{tabular}

formigas mortas e de frações de folhas vegetais íntegras e em decomposição, carreados para seu interior por certas espécies de formigas que nidificam na parte superior dos internódios (acima da água). As formigas (Formicidae), mais freqüentemente presentes em tais habitats, foram: Camponotus (Tanaemyrmex) sp. (Formicinae); Crematogaster sp. e Pheidole sp. (Myrmicinae); Azteca sp., Iridomyrmex sp., Tapinoma sp. e Tapinoma atriceps Emery,1887 (Dolichoderinae); Heteroponera inermis (Emery, 1894) e Pachycondila crenata (Roger, 1861) (Ponerinae). Já nos internódios-armadilhas, o sedimento resultou de fragmentos de insetos mortos e, também, de folhas caducas. Em superposições de espécies, o sedimento fino e os diversos detritos funcionaram como anteparo de proteção às larvas-presas.

A entomofauna culicidiana dendrotelmata, apesar de se procriar em habitats formados por recipientes fechados, isolados do meio ambiente e protegidos de inimigos externos, tem seus predadores coespecíficos e específicos que exercem certa ação controladora no tamanho destas populações.

A decomposição da matéria orgânica, em alto teor, confere à água destes 
microhabitats um odor fortemente desagradável. Nestas condições, colonizaram Culex (Carrollia) iridescens (Lutz, 1905), Cx. (Carrollia) kompi Valencia, 1973, Cx. soperi, Shannoniana fluviatilis (Theobald, 1903), To. bambusicola, To. pusillus, Trichoprosopon (Trichoprosopon) compressum var. mogilasium Dyar \& Knab,1907, Tr. (Trichoprosopon) pallidiventer (Lutz \& Neiva, 1905), Wyeomyia limai, Wy. lutzi e Wy. personata que, por sua vez, também ocorreram em águas límpidas. Estas onze populações englobam a fauna culicidiana dotada de capacidade, por composição genética e firmada ao longo de sua evolução, para obter equilíbrio na procriação em condições ecológicas adversas (LAROCA, 1995). Estes aspectos sugerem que as onze espécies, ao longo de sua evolução, tornaram-se capazes de se adaptar e de procriar em condições ecológicas amplamente diversificadas, inclusive em adversas (k-estrategistas). Ao contrário, Culex (Microculex) elongatus Rozeboom \& Lane, 1950, Cx. neglectus, Sabethes (Peytonulus) aurescens (Lutz, 1905), Sa. (Sabethes) batesi Lane \& Cerqueira 1942, Sa. (Sabethinus) melanonynphe (Dyar, 1924) e Tr. (Trichoprosopon) soaresi Lane \& Cerqueira, 1942 colonizaram microhabitats de água límpida, com pouco sedimento, sem a presença de matéria orgânica decomposta. Estas seis espécies constituem um grupo de culicídeos dendrícolas de pequena valência ecológica na escolha de habitats para procriar. Tr. soaresi é a mais vulnerável. Além de só ocorrer esporadicamente nestes microhabitats, é muito difícil obter adultos em laboratório. Seus imaturos têm exúvias muito delicadas e frágeis que, em questão de minutos após a muda das larvas, se desfazem totalmente na água. Talvez por estas razões, as larvas deste culicídeo não tenham sido descritas ainda.

Com relação à freqüência relativa das 17 espécies de mosquitos em ambos os microhabitats (tab. II), verificou-se que a colonização de internódios-verdes foi muito diversificada. Para treze espécies, os internódios de taquara perfilados verticalmente em colmos verdes, de diferentes padrões e dimensões de furos, foram os preferidos em $60,1 \%$, em contraposição a 39,9\% de internódios-armadilhas, colonizados também por treze espécies.

Considerando somente os internódios-verdes, a preferência da colonização, em ordem decrescente, de $24,2 \%$ coube aos internódios de um furo quadrado ou retangular, ao mesmo tempo que em internódios de um furo circular diminuto baixou para 19,2\%. A outros três tipos de furos coube a preferência de 8,1\%,4,7\% e 3,9\%, respectivamente (tab. II). Analisando comparativamente o conjunto de todas as espécies, a preferência por padrão de furo em internódios-verdes variou de $11,2 \%$ a $100,0 \%$. $C x$. neglectus, Cx. soperi, Sa. batesi e Sa. melanonynphe restringiram sua freqüência exclusivamente a estes microhabitats em 100,0\%. Em internódios-armadilhas, a preferência manteve-se entre 6,8 a 100,0\%. Quatro espécies atingiram o máximo de presença nestes microhabitats: Cx. elongatus, Cx. iridescens, Cx. kompi e Tr. soaresi (tab. II). O fato de se concentrarem em $100 \%$ exclusivamente em um destes tipos de microhabitats, não lhes confere extraordinária presença média por internódio.

No que tange à preferência por padrão de furo, em internódios inseridos em colmos verdes, no conjunto geral, treze espécies colonizaram só os internódios verdes de um furo quadrado ou retangular e somaram $40,3 \%$ do total. Oito espécies, que se procriaram em internódios de furo circular diminuto, obtiveram um percentual de $31,9 \%$. Estes dois tipos de microhabitats foram os mais produtivos, pois somaram um total de $72,2 \%$. Os outros três padrões de furos apresentaram as seguintes freqüências: $13,5 \%$, 
$7,8 \%$ e $6,5 \%$, perfazendo $27,8 \%$, completando assim a totalidade de $100 \%$ (tab. II). Os internódios dotados de um furo, independentemente de suas dimensões e do número de espécies, foram os mais produtivos na procriação de culicídeos dendrícolas.

Para avaliar a frequiência relativa de mosquitos de acordo com o padrão de furos em internódios de taquara verde, estabeleceu-se o critério de considerar a presença de culicídeos a partir de $20 \%$ para mais. Foram assim distribuídos: em primeiro lugar, destacaram-se os internódios de um furo (quadrado ou retangular) com a presença das seguintes sete espécies: Cx. neglectus 26,1\%, Sa. melanonynphe 28,2\%, To. pusillus 28,8\%, Cx. soperi $31,1 \%$, Wy. personata 41,4\%, Sa. aurescens $44,9 \%$ e Wy. limai, 45,7\%. Em segundo lugar, sobressaíram os internódios de um furo circular diminuto com quatro espécies: Wy. lutzi 20,8\%, Sa. aurescens 37,6\%, Sa. batesi 50\% e Sa. melanonynphe $64,1 \%$. O terceiro lugar coube aos internódios de dois furos circulares (um diminuto e outro mais amplo) com três espécies: To. pusillus 22,0\%, Wy. personata 22,6\% e To. bambusicola 45,3\%. Em quarto, apareceram os internódios de três furos (dois circulares e um quadrado ou retangular) com três espécies: $W y$. lutzi 28,1\%, Cx. neglectus $39,1 \%$ e Cx. soperi $41,4 \%$. Os internódios de dois furos, (um circular diminuto e outro quadrado ou retangular) ocuparam o último lugar com quatro espécies: $\mathrm{W} y$. lutzi $21,1 \%$, Sb. batesi $23,1 \%, C x$. soperi $27,5 \%$ e $C x$. neglectus 34,8 . Nesta última categoria, nenhuma espécie de mosquitos atingiu acima de $40 \%$ (tab. II). Confrontando, ainda, o conjunto de todas

Tabela II. Frequiência relativa comparada entre espécies de mosquitos em internódios de taquara verde* abatidos de colmos com diferentes padrões e dimensões de furos e em internódios-armadilhas** com corte transversal, instalados artificialmente na Mata Atlântica, Serra do Mar, Paraná, Brasil, período de janeiro 1985 a dezembro 1986. (c, furos circulares de 40-50 mm em internódios-armadilhas; em internódios de taquara-verde: cp, furos circulares de $2 \mathrm{~mm}$; cg, furos circulares de $5-8 \mathrm{~mm}$; q, furos quadrados de $5 \times 5 \mathrm{~mm}$ a $10 \times 10 \mathrm{~mm}$; r, furos retangulares de $8 \times 15 \mathrm{~mm}$ a $25 \times 40 \mathrm{~mm}$; p, larvas predadoras ou canibais).

\begin{tabular}{|c|c|c|c|c|c|c|c|}
\hline \multirow{3}{*}{$\begin{array}{l}\text { Combinação de padrões } \\
\text { Dimensões de furos }\end{array}$} & \multirow{3}{*}{$* \mathrm{cp}$} & \multirow{3}{*}{$\begin{array}{l}*_{\mathrm{cp}} \\
*_{\mathrm{cg}}\end{array}$} & \multirow{3}{*}{$\begin{array}{l}* \mathrm{cp} \\
* \mathrm{q}\end{array}$} & \multirow{3}{*}{$\begin{array}{c}* \text { cp- cg } \\
* \mathrm{q} \\
* \mathrm{r}\end{array}$} & \multirow{3}{*}{$\begin{array}{l}* \mathrm{q} \\
* \mathrm{r} \\
* \mathrm{r}\end{array}$} & \multicolumn{2}{|c|}{ ** int. armadilhas } \\
\hline & & & & & & total & com corte \\
\hline & & & & & & & transversal \\
\hline$\%$ de espécie por tipo de furo & $\%$ & $\%$ & $\%$ & $\%$ & $\%$ & $\%$ & $\%$ \\
\hline Culex elongatus & 0,0 & 0,0 & 0,0 & 0,0 & 0,0 & 0,0 & 100,0 \\
\hline$C x$. iridescens & 0,0 & 0,0 & 0,0 & 0,0 & 0,0 & 0,0 & 100,0 \\
\hline Cx. kompi & 0,0 & 0,0 & 0,0 & 0,0 & 0,0 & 0,0 & 100,0 \\
\hline Cx. neglectus & 0,0 & 0,0 & 34,8 & 39,1 & 26,1 & 100,0 & 0,0 \\
\hline Cx. soperi & 0,0 & 0,0 & 27,5 & 41,4 & 31,1 & 100,0 & 0,0 \\
\hline Sabethes aurescens $(\mathrm{p})$ & 37,6 & 5,9 & 1,5 & 1,0 & 44,9 & 90,9 & 9,1 \\
\hline Sa. batesi (p) & 50,0 & 7,7 & 23,1 & 11,5 & 7,7 & 100,0 & 0,0 \\
\hline Sa. melanonynphe $(\mathrm{p})$ & 64,1 & 5,6 & 1,2 & 0,9 & 28,2 & 100,0 & 0,0 \\
\hline Shannoniana fluviatilis & 5,4 & 3,9 & 2,5 & 1,0 & 2,1 & 14,9 & 85,1 \\
\hline Toxorhynchites bambusicola (p) & 0,0 & 45,3 & 5,0 & 15,7 & 16,4 & 82,4 & 17,6 \\
\hline To. pusillus (p) & 0,0 & 22,0 & 5,1 & 10,2 & 28,8 & 66,1 & 33,9 \\
\hline Trichoprosopon compressum (p) & 0,0 & 4,1 & 4,1 & 0,0 & 3,0 & 11,2 & 88,8 \\
\hline Tr. pallidiventer $(\mathrm{p})$ & 5,4 & 0,3 & 0,4 & 1,1 & 6,2 & 13,4 & 86,6 \\
\hline Tr. soaresi $(\mathrm{p})$ & 0,0 & 0,0 & 0,0 & 0,0 & 0,0 & 0,0 & 100,0 \\
\hline Wyeomyia limai & 12,4 & 17,4 & 6,0 & 11,0 & 45,7 & 92,5 & 7,5 \\
\hline Wy. lutzi & 20,8 & 11,5 & 21,1 & 28,1 & 11,7 & 93,2 & 6,8 \\
\hline Wy. personata & 19,0 & 22,6 & 4,4 & 3,8 & 41,4 & 91,2 & 8,8 \\
\hline Percentagem / padrão de furo* & 31,9 & 13,5 & 6,5 & 7,8 & 40,3 & 100,0 & 100,0 \\
\hline Percentagem ambos os habitats & 19,2 & 8,1 & 3,9 & 4,7 & 24,2 & 60,1 & 39,9 \\
\hline
\end{tabular}


as espécies, a preferência somada por padrão de furo em internódios-verdes converge ao mesmo resultado. A ordem de preferência em primeiro lugar cabe aos internódios de furo quadrado ou retangular (40,3\%); em segundo, aos de furo circular diminuto $(31,9 \%)$; em terceiro, aos de dois furos circulares (um diminuto e outro mais amplo) $(13,5 \%)$; em quarto, aos de três combinações de furos $(7,8 \%)$ e, por último, aos de duas combinações de furos, (circular reduzido e quadrado ou retangular) (6,5\%). Se ainda for levado em conta a porcentagem de ambos os habitats, a ordem de preferência nos internódiosverdes por padrão de furo continuará não alterada: $24,2 \%-19,2 \%-8,1 \%-4,7 \%$ e 3,9\% (tab. II).

Os colmos verdes, hidrobiótopos dendrotelmatas fechados com reduzido volume de água ou sem ela, diferem muito dos existentes no solo, tanto em natureza do substrato, como no tipo de água.

As populações de culicídeos, que localizam tais criadouros, dotadas de mecanismos fisiológicos semelhantes, adaptam-se às variações climáticas similares, mas com exigências diferentes frente às condições ecológicas e mesológicas do habitat. São espécies que se adaptam a grandes variações de $\mathrm{pH}$ da água. Em internódios-verdes, os valores extremos de pH oscilaram na faixa de 3,5 a 7,1. Em internódios-armadilhas, a variação dos valores extremos oscilou entre 4,5 e 8,3. A variação mais acentuada foi em colmos verdes, oscilando de fortemente ácido a neutro, enquanto em internódios-armadilhas, as cifras mantiveram-se de ácido a levemente básico. Consequentemente, observou-se sua influência no comportamento de mosquitos, em que a entomofauna de culicídeos dendrícolas apresentou maior número de exemplares na faixa do $\mathrm{pH}$ mais ácido. Isto indicaria que esta entomofauna adaptou-se melhor às variações de $\mathrm{pH}$ da água na gama de fortemente ácido a levemente básico. Segundo MARGALEF (1979), os extremos inferiores a 3,0 e superiores a 9,0 raramente ocorrem na natureza. Entretanto, há flora e fauna características nestas condições.

Destaca-se que as variações térmicas da água em internódios de colmos verdes, na presente pesquisa, mantiveram-se entre $0^{\circ} \mathrm{C}$ e $22^{\circ} \mathrm{C}$, ao passo que em internódios instalados, os gradientes térmicos oscilaram entre $2^{\circ} \mathrm{C}$ e $26^{\circ} \mathrm{C}$. A entomofauna de mosquitos estudados adaptou-se bem aos gradientes de temperatura entre $2^{\circ} \mathrm{C}$ e $24^{\circ} \mathrm{C}$. Nos meses de inverno (junho e julho), houve congelamento da água em internódios a pouca altura do chão.

As temperaturas extremas de $0^{\circ} \mathrm{C}$ e acima de $25^{\circ} \mathrm{C}$ foram fatais para as populações de mosquitos nos recipientes estudados. Nestas circunstâncias, a mortalidade de larvas e pupas foi total. Na Inglaterra, durante o rigoroso inverno com temperaturas negativas, BRADSHAW \& HolzAPFEL (1991) conferiram a sobrevivência de três espécies: Aedes geniculatus (Olivier, 1791), Anopheles plumbeus Stephens, 1828 e Culex torrentium Martini, 1925 em águas congeladas de ocos de árvores. Conforme MArGalef (1979), em baixas temperaturas, o glicerol dissolvido na hemolinfa exerce importante função protetora nos insetos. Pelo visto, na Serra do Mar, as larvas de culicídeos tiveram menor concentração de glicerol por peso corporal que as encontradas na Inglaterra, uma vez que todas sucumbiram na temperatura de $0^{\circ} \mathrm{C}$. Uma determinada concentração de glicerol faz baixar o ponto de solidificação. CoPELAND \& CRAIG (1990), nos EUA, na região de Great Lakes, ao estudarem Anopheles barberi Coquilllett,1903, Orthopodomyia alba Baker, 1936 e Orthopodomyia signifera Coquillett, 1896 em ocos de árvores em diferentes gradientes de temperaturas negativas durante o inverno, também constataram que as 
três espécies passam o intenso frio em diapausa. A entomofauna de mosquitos brasileira parece não possuir este recurso genético, pois na presente investigação, durante o inverno houve congelamento da água e conseqüente morte de larvas e pupas nos internódios próximos ao solo, embora tenha sido preservada a vitalidade dos ovos de diversas espécies na temperatura de $0^{\circ} \mathrm{C}$. Temperaturas abaixo de zero não foram constatadas na área estudada.

Pesquisar em habitats dendrotelmatas, como os internódios de taquara e os ocos de árvores, dificulta a coleta de imaturos devido às estratificações verticais de difícil acesso. Os internódios, por sua vez, impedem o acesso às amostras por serem recipientes fechados. As investigações nestes microhabitats são escassas e fornecem informações limitadas. Os culicídeos, que selecionam internódios de taquara verde, concretizam sua façanha graças à interferência da fauna associada. As espécies Eucalyptra barbara Schaus, 1894 e Eucalyptra fumida Schaus, 1894 (Lepidoptera, Noctuidae), premidas pelas necessidades bionômicas, elaboram furos circulares nas paredes laterais dos internódios de taquara e assim possibilitam o acesso às fêmeas para a oviposição (LozovEI, 1998). Os intervenientes dos furos quadrados e retangulares não foram flagrados nem identificados. Já o acesso de mosquitos aos internódios-armadilhas foi facilitado pela interferência humana. Portanto, a sobrevivência ao longo dos anos destes mosquitos dendrotelmatas revela as peculiaridades mesológicas e bionômicas que se integram na ação conjunta da flora (taquara), fauna ( formigas, lepidópteros, além de outra fauna não identificada) e muitas vezes o concurso da natureza, como o vento ao quebrar colmos de taquara e a interferência humana, mesmo involuntária e abrem as portas de entrada (furos) às fêmeas para a oviposição.

Agradecimentos. À Dra. Maria Anice Mureb Sallum, Departamento de Epidemiologia, Universidade de São Paulo, São Paulo, pelo auxílio na identificação das espécies de mosquitos. Ao Dr. Carlos Roberto Ferreira Brandão (MZSP), pela gentileza de identificar os espécimens de formigas.

\section{REFERÊNCIAS BIBLIOGRÁFICAS}

Amerasinghe, F. P. \& Alagoda, T. S. B. 1984. Mosquito oviposition in bamboo traps, with special reference to Aedes albopictus, Aedes novalbopictus and Armigeres subalbatus. Insect Sci. Applic., Nairobi, 5 (6): $493-$ 500.

Bradshaw, W. E. \& Holzapfel, C. M. 1991. Fitness and habitat segregation of British tree-hole mosquitoes. Ecol. Entomol., Oxford, 16: 133-144.

Copeland, R. S. \& Craig, G. B. Jr. 1990. Cold hardiness of tree-hole mosquitoes in the Great Lakes region of the United States. Can. J. Zool., Ottawa, 68: 1307-1314.

Forattini, O. P. 1965a. Entomologia Médica. São Paulo, Universidade de São Paulo. v. 3, 506 p. 1965b. Entomologia Médica. São Paulo, Universidade de São Paulo. v. 2, 416 p.

Gomes, A. C.; Forattini, O. P. et al. 1992. Microhabitats de Aedes albopictus (Skuse) na região do Vale do Paraíba, Estado de São Paulo, Brasil. Revta Saúde públ., São Paulo, 26 (2): 108-118.

LanE, J. 1953a. Neotropical Culicidae. São Paulo, Universidade de São Paulo. 548 p. 1953b. Neotropical Culicidae. São Paulo, Universidade de São Paulo. 1112 p.

Laroca, S. 1995. Ecologia: Princípios e Métodos. Vozes, Petrópolis. 197 p.

LOPES, J. 1997. Ecologia de mosquitos (Diptera: Culicidae) em criadouros naturais e artificiais de área rural no Norte do Estado do Paraná, Brasil. V. Coleta de larvas em recipientes artificiais instalados em mata ciliar.Revta Saúde públ., São Paulo, 31 (4): 370-377.

Lozover, A. L 1998. Mosquitos dendrícolas (Diptera, Culicidae) em internódios de taquara da Floresta Atlântica, Serra do Mar e do Primeiro Planalto, Paraná, Brasil. Brazilian Arch. Biol. Technol., Curitiba, 41 (4): 501 508 . 
Lozover, A. L. \& Luz, E. 1976. Diptera Culicidae em Curitiba e Arredores. I. Ocorrência. Arq. Biol. Tecnol., Curitiba, 19: 25-42.

MAACK, R. 1981. Geografia Física do Estado do Paraná. 2ª ed., Rio de Janeiro, J. Olympio. 450 p.

MARGAlef, R. 1974. Ecologia. Barcelona, Omega. 951 p.

MiYAGI, I. 1976. Description of a new species of the genus Topomyia Leicester from the Ryukyu Islands, Japan. Trop. Med., Nagasaki, 17: 201-210.

Mogi, M. 1978. Intra- and intespecific predation among filter feeding mosquito larvae. Trop. Med., Nagasaki, 20: $15-27$.

Mogi, M. \& SuzuKI, H. 1983. The biotic community in the water-filled internode of bamboos in Nagasaki, Japan, with special reference to mosquito ecology. Jap. J. Ecol., Tokyo, 33 (3): 271-279.

MoRIYA, K. 1974. Seasonal trends of field population of mosquitoes with ovitramps in Kanagawa Prefecture. 1. Comparison of the populations of four residential areas in Kamakura City in 1971. Jap. J. Sanit. Zool., Tokyo, 25: 237-244.

Nakata, G.; Matsuo, L. \& Ito, S. 1953. Ecological studies on mosquitoes about Kyoto City (1). On the succession of mosquito larvae breeding in minute inland waters found in graveyards and bamboo groves. Jap. J. Sanit. Zool., Tokyo, 4: 62-71.

Service, M. W. 1976. Mosquito Ecology. Field Sampling Methods. London. Applied Science. 583 p.

Silva, M. A. N. \& LozoveI, A. L. 1999. Ocorrência de Haemagogus (Conopostegus) leucocelaenus (Dyar \& Shannon, 1924) e Toxorhynchites (Lynchiella) theobaldi (Dyar \& Knab, 1906) em ocos de árvore, em capão de mata, Curitiba, Paraná, Brasil. Revta bras. Zool., Curitiba, 16 (Supl. 1): 257-267

WALKER, E. D.; LAWSON, D. L. et al. 1991. Nutrient dynamics, bacterial populations, and mosquito productivity in tree hole ecossystems and microcosms. Ecology, Brooklyn, 72 (5): 1529-154

Recebido em 07.06.1999; aceito em 26.06.2000. 